\title{
Bayesian Network Models for Making Maintenance Decisions from Data and Expert Judgment
}

\author{
Haoyuan Zhang \& D. William R. Marsh \\ Risk and Information Management Research Group, School of Electronic Engineering and Computer \\ Science, Queen Mary University of London
}

ABSTRACT: To maximize asset reliability cost-effectively, maintenance should be scheduled based on the likely deterioration of an asset. A number of types of statistical model have been proposed for predicting this but they have important practical limitations. We present a Bayesian network model that can be used for maintenance decision support to overcome these limitations. The model extends an existing statistical model of asset deterioration, but shows how i) failure data from related groups of asset can be combined, ii) data on the condition of assets available from their periodic inspection can be used iii) expert knowledge of the causes deterioration can be combined with statistical data to adjust predictions and iv) the uncertain effects of maintenance actions can be modelled. We show how the model could be used for a range of decision problems, given typical data likely to be available in practice.

\section{INTRODUCTION}

\subsection{Maintenance decision making}

Maintenance consists of a set of activities required to ensure assets are in a reliable operating condition (Pintelon \& Gelders, 1992). According to Dhillon (2002), maintenance work can be of three types: i) corrective maintenance, where maintenance follows failure ii) preventive maintenance, where inspections and maintenance follow a fixed schedule and iii) predictive maintenance, where the schedule depends on the condition of assets. Predictive maintenance requires a way to predict the future condition of an asset by estimating how fast it will deteriorate from its current condition.

A range of statistical models, using Weibull, gamma or exponential distributions, has been used to model deterioration (see section 2). However, in many situations these do not provide practical decision tools as the assumed data is unavailable and relevant knowledge (such as use and environment) that could be used to distinguish between different individuals in the same asset class is unused.

This paper proposes an asset deterioration model building on an existing statistical model that can be adapted to exploit the available data and incorporate expert judgment on factors that affect the likely deterioration rates. We show how to use the model for practical decision-making, including the effect of different maintenance actions.

\subsection{Paper outline}

Section 2 reviews existing deterioration models and establishes what is needed for maintenance decisionmaking in practice. Section 3 introduces a sequence of Bayesian network (BN) models to overcome the practical limitations of existing models and Section
4 gives an example application of the $\mathrm{BN}$ models. Conclusions are in Section 5.

\section{EXISTING DETERIORATION MODELS}

\subsection{Models for asset maintenance decision making}

In time-based maintenance, the expected lifetime of an asset is derived from its likely time to failure. A set of failure time data is gathered and fitted to a statistical distribution, describing the failure probability of an asset at a given time. Many distributions have been used: for example Guler et al. (2011) and $\mathrm{He}$ et al. (2013) use exponential distributions to estimate degradation of railway track. The gamma distribution, in the form of gamma processes, is used by Edirisinghe et al. (2012) to study the deterioration of building components.

However, the Weibull distribution, with probability density over time $t$ shown in equation 1 , is the most popular distribution for this purpose as it can describe a range of deterioration behaviours.

$$
f(t)=\frac{\beta}{\eta}\left(\frac{t}{\eta}\right)^{\beta-1} e^{-\left(\frac{t}{\eta}\right)^{\beta}}, f(t) \geq 0, \beta \geq 0, \eta \geq 0
$$

The two parameters of the distribution are scale $\eta$ and shape $\beta$. For a given shape, increasing the scale increases the mean failure time. The effect of the shape parameter on the failure rate is shown in Figure 1, with shape $<1$ describing early degradation leading to decreasing failure rate and shape $>1$ describing wear-out failure, giving an increasing failure rate. Bridges (Agrawal et al., 2009) and railways (Andrews, 2012) are two examples showing the use of Weibull to model a range of asset deterioration behaviours. 
Failure rate function of Weibull distribution $($ scale $=5)$

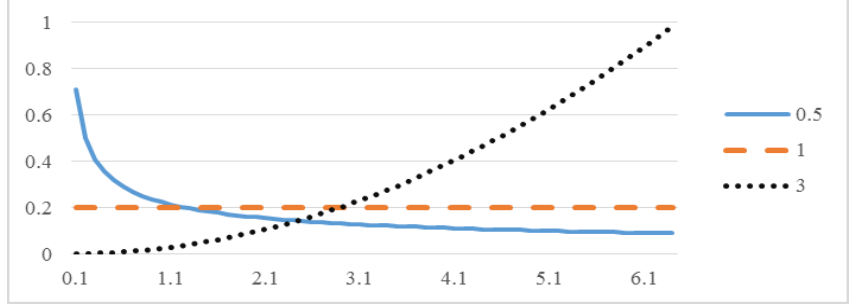

Figure 1. Failure rate function with different shape values.

Markov models have been proposed as an alternative to time-based statistical models, especially for applications that distinguish several states of repair. Deterioration is modelled by a sequence of states with, in simple models, a fixed transition probability from one state to the next. Micevski et al. (2002) use Markov models for deterioration of storm water pipes. Jiang et al. (1988) and Cesare et al. (1992) apply Markov models to predict future state of bridges, and Shafahi \& Hakhamaneshi (2009) used it for track maintenance prediction.

Deterioration models that combine several repair states with changing transition probabilities have been implemented using Petri nets and Monte-Carlo simulation. Audley \& Andrews (2013) used Petri net to model the degradation of track, developing an optimum inspection and maintenance policy. A hierarchical Petri net model for rail track maintenance is presented by Rama \& Andrews (2013), but the data needed to parameterise the model is not described in detail.

\subsection{Practical decision in maintenance}

Models for managing asset maintenance combine a deterioration model with a decision model (Frangopol et al., 2004). Statistical models can model the different aging processes of assets but directly distinguish only working from (hard) failure (Gorjian et al., 2010); this is not sufficient for using inspection data and making decisions about a variety of maintenance actions, for which Markov models with multiple states of repair - are more suited.

The availability of sufficient data is also a concern: we may only have data such as a history of repairs, from which the condition of assets could be inferred with considerable uncertainty. Further, separate statistical models are created from failure data of each different type of asset: a difficulty for an organisation with many different asset types but only a few of each. We would therefore like to be able to pool failure data where we have evidence that different but related asset types have related aging processes.

Decisions about maintenance can be made for specific structure, whereas statistical models cover all asset of the same type. It should be possible to use experience (e.g. among maintainers) about the effect of factors such as environmental conditions, loading and design differences on the rates of deterioration in combination with models derived from failure data in a population where these factors vary.

\section{BAYESIAN MODELS OF ASSET CONDITION}

A Bayesian network (BN) represents the joint probability of a set of random variables; causal or influential relationships between variables are specified by a directed graph with the variables as nodes. Additionally, the $\mathrm{BN}$ is parameterized by a conditional probability distribution for each variable, depending on the states of its parents. When some variables have a known state, an inference algorithm can update the probability distribution of the remaining variables, using Bayes' theorem. A BN can be created in a variety of ways, including from a combination of statistical data and expert opinion.

Early, easy-to-use BN inference algorithms worked primarily with discrete variables, allowing continuous variables only by discretisation. This was a barrier for the use of BNs in reliability analysis where both discrete and continuous variables are needed. Neil et al. (2007) presented a hybrid BN that uses an inference algorithm where continuous variables are dynamically discretised, with narrower intervals where the probability distributions are changing most. This algorithm is implemented in the the BN tool AgenaRisk (Agena Ltd, 2007). Marquez et al. (2007) applied this algorithm to model continous failure times of components and overall system reliability.

In this paper we present a hybrid $\mathrm{BN}$ model to support maintenance decision making, where assets deterioration follows a Weibull distributions. Section 3.1 presents a Bayesian network incorporating an existing statistical model of failure, learnt from data. Section 3.2 extends this to transition ages between asset conditions and Section 3.3 shows how to pool data from different asset types with similar deterioration rate. Section 3.4 demonstrates the capability of the model to use more realistic types of failure data. Section 3.5 extends the model to include expert judgment to customize the prediction for a particular asset. Section 3.6 shows how maintenance actions can be added to the model.

\subsection{Parameters learning using Weibull distribution}

In Marquez et al. (2007), a hierarchical BN was used to compute the unknown failure rate of an asset using historical data gathered from assets assumed to have similar failure behaviour. The $\mathrm{BN}$ is called hierarchical as the unknown parameters of the statistical distribution are modelled as probabilistic 
variables (called hyper-parameters), allowing both parameter learning and prediction in the same model.

To construct the hierarchical failure model, prior probability distributions of hyper-parameters, such as the Weibull's shape and scale, need to be assigned. Prior distributions of shape and scale are independent as no joint conjugate prior is available when they are both unknown.

It is difficult for non-statisticians to evaluate the values of shape and scale. But, by understanding the characteristics of the hyper-parameters, it is easier for experts to justify whether a type of asset has a decreasing, constant or increasing failure rate, leading to a range of possible values for the shape parameter. Similarly, it is also possible to evaluate the range of the scale parameter from the typical age of asset failure. Different distributions, such as normal distribution or uniform distribution, could be used to express uncertainty over the range of each parameter. Followed Marquez et al. (2007), triangular distributions (equation 2) are used in our model:

$$
\beta, \eta \sim \text { Triangular }(a, b, c)
$$

where $a$ is the lower limit, $b$ is the median and $c$ is the upper limit.

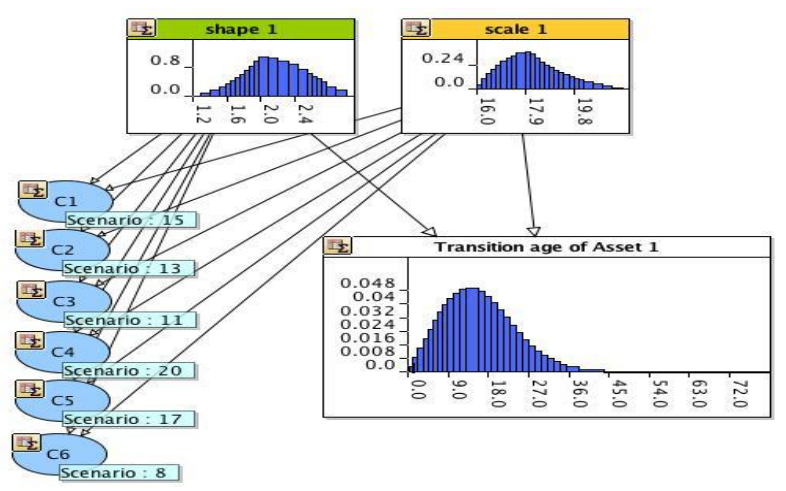

Figure 2. Parameters learning using Weibull distribution.

Figure 2 presents the $\mathrm{BN}$ model constructed on these principles. The age each asset transitions from a normal to a failed state follows a Weibull distribution, where their shape and scale are governed by the hyper-parameters shapel and scale1, whose prior probability distributions are specified using triangular distributions. Data on the past transition ages with a mean value of 14 are entered as evidence. The posterior distribution of transition age, which predicts future failure, is shown for the variable Transition age of Asset 1, derived from the hyper-parameters learnt from data.

We only show failure data for six assets in the figure. However, increasing the size of the dataset causes the parameters of the Weibull distribution to start to converge. This indicates that with sufficient failure data, it is possible to overcome experts' judgments on the range of the hyper-parameters. The tradeoff between the influence of experts and data require further study.

\subsection{Prediction of asset condition}

To model the deterioration of assets through several states of repair, we adopt the grading system used in Le \& Andrews (2015), where the condition of assets is classified as: New, Good, Poor and Very Poor.

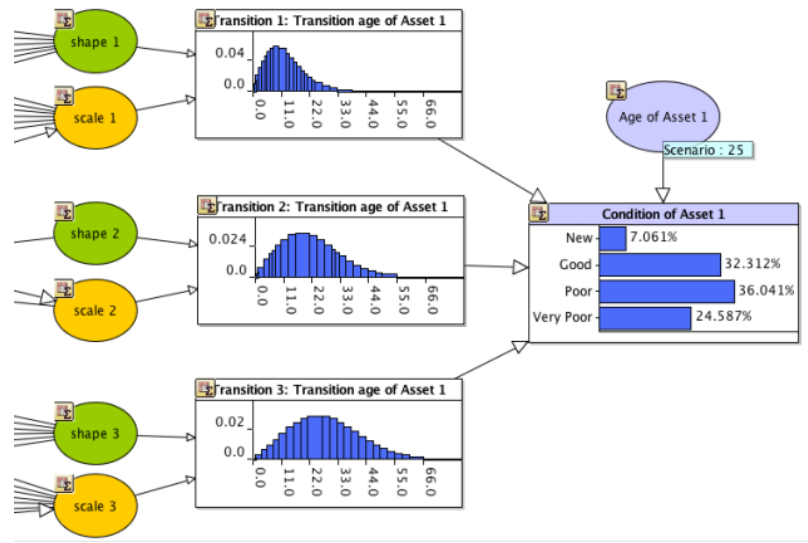

Figure 3. Prediction of asset condition.

By using the model in section 3.1, we can extend it with multiple states (Figure 3), with transition ages from New to Good (Transition 1), Good to Poor (Transition 2), and Poor to Very Poor (Transition 3), each modeled by Weibull distributions.

When decision makers enter the age of an asset, the asset condition will be calculated using the following Boolean logic expression:

$$
\begin{aligned}
& \text { if }\left(\text { Age }<\mathrm{T}_{\mathrm{N} \rightarrow \mathrm{G}},\right. \text { "New", } \\
& \text { if }\left(\text { Age }<\mathrm{T}_{G \rightarrow \mathrm{P}},\right. \text { "Good", } \\
& \text { if }\left(\text { Age }<\mathrm{T}_{P \rightarrow \mathrm{V}},\right. \text { "Poor", "Very Poor" )) }
\end{aligned}
$$

where:

- Age is Age of Asset;

- $\mathrm{T}_{\mathrm{N} \rightarrow \mathrm{G}}, \mathrm{T}_{\mathrm{G} \rightarrow \mathrm{P}}$ and $\mathrm{T}_{\mathrm{P} \rightarrow \mathrm{V}}$ are Transition 1, Transition 2 and Transition 3 respectively.

Therefore, we can capture the condition distribution of the asset based on its age. This variable can be used by decision makers to evaluate if an inspection needs to be carried out.

\subsection{Assets with similar deterioration rate}

In practice, we may have several groups of assets of different types, which we believe deteriorate with similar behaviour. We propose to extend the model in Section 3.2 for the situation where there is a lot of failure data for some types of assets in the group, but much less for others. We assume the distribution hyper-parameters learnt for one type asset approximate those of the other type. 


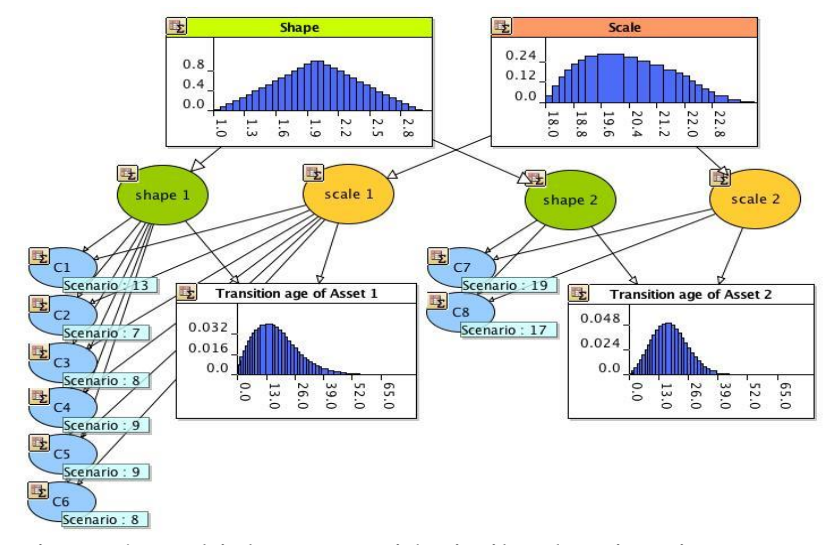

Figure 4. Multiple assets with similar deterioration.

Assume Asset 1 and Asset 2 have similar deterioration rates resulting from some shared characteristics. Figure 4 presents a parameters learning model for these two assets. Shapes (node shape 1 and shape 2) and scales (node scale 1 and scale 2) of these assets were governed by the typical shape (node shape) and scale (node scale) variables, given prior probability distributions as before (Section 3.1). A truncated normal (TNormal) distribution, a normal distribution bounded by lower and upper limits, is used to model the relationship between the related shape and scale of each asset type and the typical ones, as following:

shape1, shape $2 \sim$ TNormal (shape, $\left.\sigma^{2}, 0,3\right)$

scale1, scale $2 \sim$ TNormal $\left(\right.$ scale $\left., \sigma^{2}, 0,50\right)$

The mean of this distribution is the typical shape range shape, and variance $\sigma^{2}$ representing the degree of similarity of the two assets, which is given by experts. The upper bounds of the distributions are also evaluated by experts. By using expert judgement about the degree of similarity of different assets, the model can reason about the transition age of an asset for which there is only a little data using data from other assets that are judged to share a similar deterioration rate.

\subsection{Modelling of available data types}

Often, the ideal data on the failure times of assets is not available. This section explores various limitations on the data likely to be available data, showing how it can be used.

Transition age, the age of asset transitions from state $i$ to another state $\mathrm{j}$, is written as $\mathrm{T}_{\mathrm{i} \rightarrow \mathrm{j}}$. Thus, $\mathrm{T}_{\mathrm{N} \rightarrow \mathrm{G}}, \mathrm{T}_{\mathrm{G} \rightarrow \mathrm{P}}$ and $\mathrm{T}_{\mathrm{P} \rightarrow \mathrm{V}}$ are transition from New to Good, Good to Poor and Poor to Very Poor respectively. In practice, we are more likely to have data from periodic inspections (and perhaps repairs) rather than data on the exact transition age. To exploit the inspection history data, four types of transition age data can be inferred with uncertainty as follows (for simplicity we assume inspection interval is 5 months, and the transition are between New to Good):

- Left-censored data: the asset failed at some point before we started to inspect. Hence, the transition age is less than 5 months: $\mathrm{T}_{\mathrm{N} \rightarrow \mathrm{G}}<5$.

- Interval-censored data: failures happened sometime between two inspection times, so the asset transitioned between 5 and 10 months, therefore, $5<\mathrm{T}_{\mathrm{N} \rightarrow \mathrm{G}} \leq 10$.

- Right-censored data: for those cases where the asset survived longer than the time available for observation. Suppose an asset has been inspected twice and has survived for more than 10 months, hence $\mathrm{T}_{\mathrm{N} \rightarrow \mathrm{G}}>10$.

- Exact-time data: this type of data may be available when an issue is reported at 8 months and inspection confirms the transition, so for example $\mathrm{T}_{\mathrm{N} \rightarrow \mathrm{G}}=8$.

To use these data, we introduce a censored boolean variable as a posterior of an asset's transition age. To represent left censored data, the variable is true when $T_{i \rightarrow j}<t_{\text {inspection }}$ and the true state is observed. Similarly constructions are used for interval and right censored data.

Furthermore, there is a possibility that a component deteriorates faster than our inspection intervals. For example, suppose that at the 5-month inspection, a component remained at New, while in the 10-month inspection, the component was found in the Poor condition. To use this type of information, we can enter observations for right censored data that its first transition age is greater than 5 months, and left censored data that its second is smaller than 10 months, as well as an additional constraint that the first transition age is smaller than the second one, as following:

$$
\left(\mathrm{T}_{\mathrm{N} \rightarrow \mathrm{G}}>5\right) \wedge\left(\mathrm{T}_{\mathrm{G} \rightarrow \mathrm{P}} \leq 10\right) \wedge\left(\mathrm{T}_{\mathrm{N} \rightarrow \mathrm{G}} \leq \mathrm{T}_{\mathrm{G} \rightarrow \mathrm{P}}\right)
$$

\subsection{Introducing experts to distinguish individual asset deterioration}

In practice, the deterioration rate may be affected by heavy use and aggressive environment conditions (see for example Kumar (2006)). Ideally, the maintainers' knowlegde of these effects could be combined with statistical failure data gathered from a population where use and enviorment vary. From a decision support perspective, this will allow specific assets to be distinguised. For example, Marsh et al. (2015) outline a BN architecture to integrate multiple factors, such as loading and environment influences, to support maintenance decision but do not show failure data could be included.

To distinguish individual members of a group of assets, we model the effect of environmental 
conditions and loading on deterioration by adjusting the scale parameter of the $\mathrm{BN}$ developed in previous sections. A known shape parameter is often assumed due to its relatively stable value (Nordman \& Meeker, 2002, Jun et al., 2006).

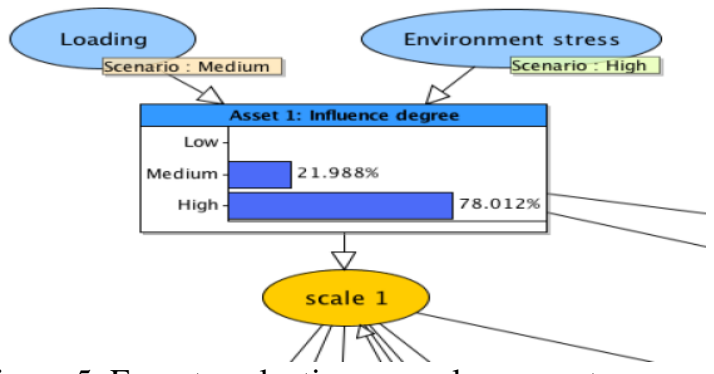

Figure 5. Expert evaluation on scale parameter.

As shown in Figure 5, we use two ranked nodes to express the degree of influence of factors (here, for example, loading and environment). These are combined using a weighted mean (wmean, equivalent to a linear model) and the 'adjusted scale' is modelled by a TNormal with mean adjusted from the scale hyper-parameter:

$$
\text { Influence degree } \sim \text { TNormal (wmean, } \sigma^{2}, O, I \text { ) }
$$

In Figure 5 we assume the weight of loading is 0.3 , environment condition is 0.7 , so that the combined influence of these factors is slightly closer to the high environment stress than the medium loading.

\subsection{Maintenance}

The BN models of the previous sections cover the distributions of transitions ages between different conditions and can therefore predict the future condition of an asset, allowing decisions about, for example, the interval to the next inspection. Here we further extend the model to include maintenance actions, supporting a wider range of decisions. The extended model shown in Figure 6, provides the following predictions:

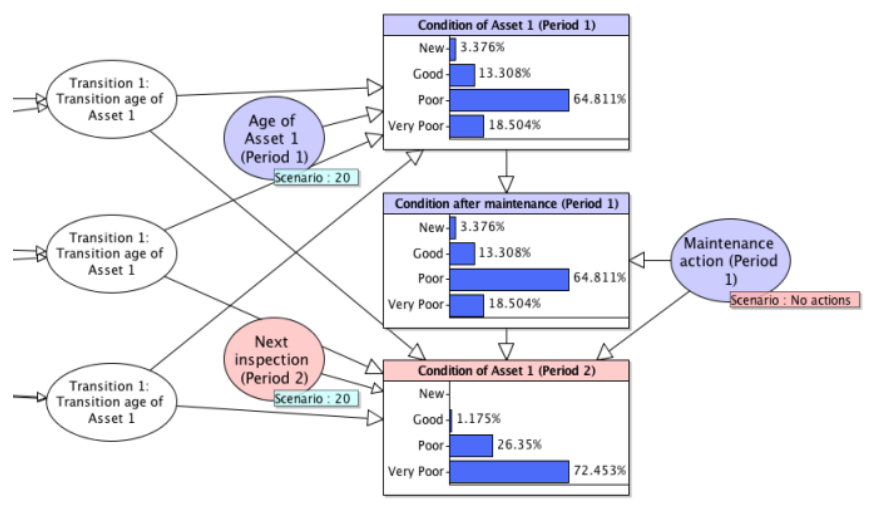

Figure 6. Maintenance.

- Condition of Asset (current period): the asset's condition based on its age (see section 3.2);
- Condition after maintenance (current period): its predicted condition after a maintenance action;

- Future condition (next inspection): to reason the future condition of the asset, taking into account both the maintenance action and further deterioration.

\subsubsection{Condition after maintenance}

In contrast with the assumption made in Le \& Andrews (2015), we do not assume maintenance can restore the asset back to its new condition completely, which would be more practical in real life applications.

Four types of maintenance scenarios are modelled: no actions, minor intervention, major intervention and renewal. The effect of each different actions can be modelled using the following principles:

- No actions: the distribution of Condition after maintenance (Period 1) will stay the same as the Condition of Asset 1 (Period 1);

- Minor intervention: restore asset in Good condition back to New condition with a successful rate of $95 \%$, but cannot cope with Poor and Very Poor condition;

- Major intervention: restore asset in Good condition back to New condition with a $100 \%$ successful rate. Also, it can restore asset in Poor condition with a $90 \%$ probability back to $\mathrm{New}$ condition, $8 \%$ back to Good condition, and 2\% of failure (stays at Poor). But it cannot cope with Very Poor condition;

- Renewal: restore asset condition (no matter what condition it was) back to New condition with a $100 \%$ successful rate.

Hence, we can predict the condition distribution after different actions were taken. By entering maintenance actions as evidences, the node can present the new condition distributions of the asset. Decision makers can use this node to estimate the effect of different actions on the uncertain asset state.

\subsubsection{Future condition}

Following maintenance, the future condition depends both on the success of the maintenance action and the further deterioration. If the state of the asset is changed following maintenance, we call the maintenance effective. The time for deterioration is then the time since the maintenance. If the maintenance is ineffective - it produces no change in condition - then the time of the asset deterioration will be the accumulation of time from last effective maintenance.

The node of Condition of Asset 1 (Period 2) is to serve the purpose of predicting future condition of 
an asset considering whether effective maintenance action was taken with the following logic:

if $(\mathrm{CM}=" \mathrm{New}$ " and

(MA =" No actions" and Age $1+$ Age $_{2}<\mathrm{T}_{\mathrm{N} \rightarrow \mathrm{G}}$ ) $\|$

(MA $\neq$ "No actions" and $\mathrm{Age}_{2}<\mathrm{T}_{\mathrm{N} \rightarrow \mathrm{G}}$ ), "New",

else if $(\mathrm{CM}=$ "Good" and

(MA =" No actions" and Age $1+$ Age $_{2}<\mathrm{T}_{\mathrm{G} \rightarrow \mathrm{P}}$ ) $\|$

(MA $\neq$ "No actions" and Age ${ }_{2}<\mathrm{T}_{\mathrm{G} \rightarrow \mathrm{P}}$ ), "Good",

else if $(\mathrm{CM}=$ "Poor" and

(MA =" No actions" ||MA ="Minor intervention"

and $\left.\mathrm{Age}_{1}+\mathrm{Age}_{2}<\mathrm{T}_{\mathrm{P} \rightarrow \mathrm{V}}\right)$

(MA =" Major intervention"||MA =" Renewal"

and Age $_{2}<\mathrm{T}_{\mathrm{P} \rightarrow \mathrm{V}}$ ), "Poor", "Very Poor")))

where:

- $\mathrm{CM}$ is the Condition after maintenance (Period 1);

- $\quad$ MA is the Maintenance actions (Period 1);

- Age $_{1}$ is Age of Asset 1 (Period 1), Age 2 is Next inspection (Period 2);

- $\mathrm{T}_{\mathrm{N} \rightarrow \mathrm{G}}, \mathrm{T}_{\mathrm{G} \rightarrow \mathrm{P}}$ and $\mathrm{T}_{\mathrm{P} \rightarrow \mathrm{V}}$ are Transition 1, Transition 2 and Transition 3 respectively.

Consequently, this node predicts an asset's condition distribution based on the selected action within different periods. Decision makers can use it to plan their maintenance actions by assessing future condition distribution based on the selected actions. stages in Section 3 can be used to support decisionmaking in asset maintenance planning. The combined model is shown in Figure 7 with the following details:

- The variables shown as rectangles are distributions (for simplicity reason, the transition age of assets are hidden); ovals are evidence variables;

- Each asset can be in one of four conditions, from New to Very Poor; this requires three transition models: Transition 1, Transition 2 and Transition 3;

- Two different assets but related are included: asset 1 , with only a little condition data, and asset 2 for which there is much more data

- $\mathrm{C} 1, \mathrm{C} 2, \ldots, \mathrm{C} 11$ are observations of deterioration for asset 1;

- Similarly, C12, C13, ..., C47 are observations of deterioration for asset 2 ;

- Expert judgement about the scale of asset 1 and asset 2 are presented in the top left block;

- Decision support about conditions of asset 1 is showed in the top right block.

\subsection{Inference from Imperfect data and Limited data}

For asset 1, we have inspection data observing the first transition (from New to Good) but little data for later transitions; asset 2 has extensive data for all transitions. To use this data, censored data variables (from section 3.4) are implemented:

- C1 stayed at New condition in its 5-month inspection, but was in condition Good by the time of its 10-month inspection. Thus, an

The section shows how the BN model developed in

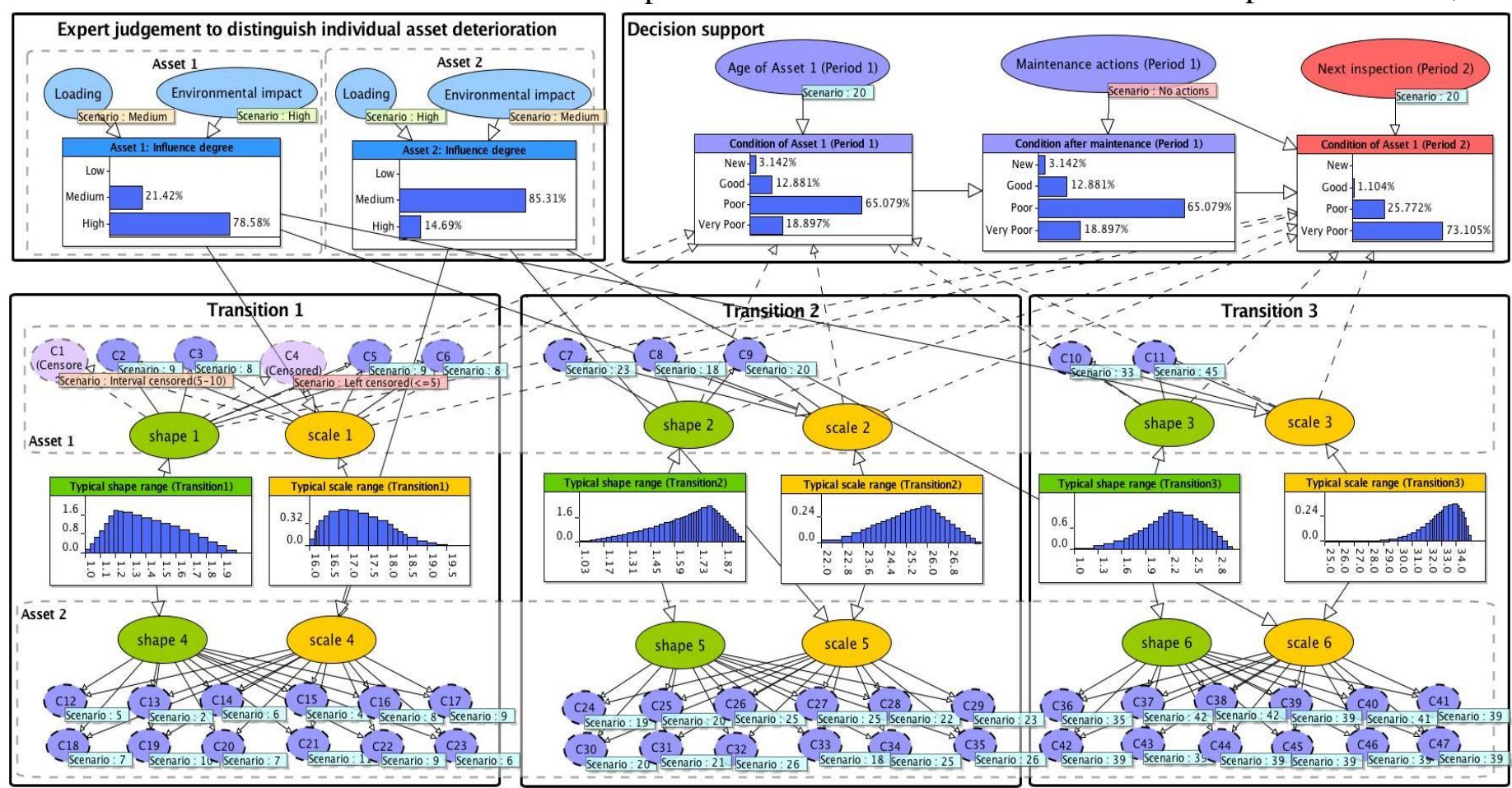

Figure 7. Maintenance decision making model. 
interval censored data variable is used and transition age between 5 and 10 months is modelled.

- C4 is a new asset; when inspected at its first 5month inspection it was already found to have started deteriorating. Hence, we modelled it as a left censored data variable, indicating a transition age smaller than 5 months;

Also, as a newly developed asset type, asset 1 does not have enough data about its condition, especially its transitions from Good to Poor and Poor to Very Poor; only three and two data are available respectively. While asset 2, an older version of asset 1, shared similar deterioration rate with asset 1, has lots of data. Two approaches, developed in section 3.3 and 3.5, are applied to learn from asset 2:

- In the middle of each transition block, typical range of shapes and scales are implemented. The means of all parameters are supervised by the typical range distributions. Variances, evaluated according to the similarity of two assets by experts, are also included in corresponding parameters;

- In the top left expert judgement block, factors that have impacts on the value of scale are considered. Compared to high loading and medium environmental condition in asset 2 , asset 1 suffered medium loading but severe environmental condition. A higher influence degree indicates an asset will deteriorate faster, which will result in a smaller scale value.

The impact of being able to use a wider range of data can be seen on the scale value, for example. Using the available perfect data only (i.e. the exact transition ages), the scale values of asset 1 are 17.9 for the first transition (Transition 1), 25.2 in Transition 2 and 30.3 in Transition 3. When the censored data and the pooled data from asset 2 (whose scale values are 13.3, 22.5 and 31.2) are used, the scale value of asset 1 changed, considering both its similarity and the external influence, with learned result of 12.0, 20.9 and 28.2 respectively.

Although more experiments are needed to evaluate its accuracy, this model proves its flexibility when dealing with imperfect and limited data.

\subsection{Maintenance Planning}

One feasible way for decision makers to make use of this model is to investigate asset condition distributions at different times, which is an essential criterion for maintenance planning. By setting a threshold of a particular state, we can provide decision makers with information about when maintenance actions are needed.

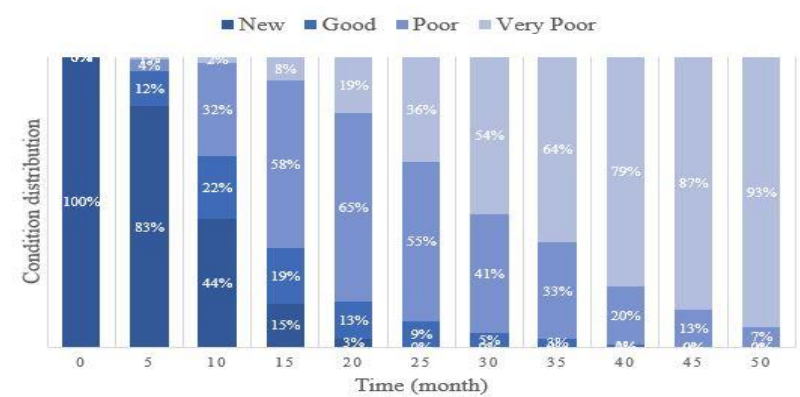

Figure 8. Condition distributions at different times.

Figure 8 shows how asset 1's predicted state changes from 0 to 50 months. The probability of New condition drops dramatically, reaching $0 \%$ after 25 months, meanwhile Good condition and Poor conditions increase at first, reaching peaks at 10 months and 20 months respectively. Very Poor condition keeps increasing over times.

Maintenance planners can set a threshold to decide when action is needed. For example, when the probability of asset 1 in Very Poor condition is greater than $30 \%$, renewal action needs to be carried out. Thus, from Figure 8, we can schedule a renewal action when it reached 25 months.

\section{CONCLUSIONS}

\subsection{Summary}

This paper presents an asset deterioration model with maintenance decision support using a Bayesian network. The core of the model uses a Weibull distribution to model asset deterioration in a hierarchical $\mathrm{BN}$. The transition times between a small number of conditions are assumed to be drawn from Weibull distributions, whose parameters are learned using historical data. To complete the hierarchical model, prior probabilities need to be assigned to the hyper-parameters. The shape parameter can model an increasing, constant or decreasing failure rate, while the scale parameter stretches out the probability density function. By understanding the characteristics of these two parameters, we argue that reasonable priors could be determined in discussion with experts.

We may have a group of related asset types that deteriorate with similar behaviour, with a lot of data for some types, while others have little. We extend the model to learn from related assets. A further expert judgement of the degree of similarity between the related assets is needed at this stage. We also extend the model to make the most use of available data, which do not necessary include the exact times that each asset transitioned from one state to another. Then, in order to distinguish individual assets within 
a broad class of similar assets, we allow experts to quantify the effect of factors such as loadings and environmental conditions to adjust the predicted deterioration of a specific asset. This expert judgment could be replaced by parameters learned from data if sufficient were available.

Finally, we show how the different modelling steps can be combined and used for maintenance decision problems. Although only a single scenario is modelled, it is sufficient to show the flexibility of the approach for a wide range of situations.

\subsection{Future Work}

Further work is needed to validate the approach using real datasets and for real maintenance scenarios. We wish to extend the model further to incorporate costs, including disruption, of different maintenance actions, so that we can schedule an optimum hierarchy of maintenance actions.

We also plan to study the computational performance of the model, especially for significant quantities of data. Experience to date shows that it is adequate and some optimisations to the model structure are likely to yield improvements. Fortunately, maintenance decision making does not require instant answers and there are a great variety of $\mathrm{BN}$ inference algorithms offering different tradeoffs between speed and accuracy.

As we have shown in Section 4, the way that the different modelling stages are combined reflects the needs of a particular scenario. To make this practical, we plan to develop a higher-level interface in which the maintenance-related information (such as the number of conditions, the maintenance actions etc.) could be described and from which the necessary $\mathrm{BN}$ could be generated automatically.

\section{REFERENCE}

Agena Ltd. 2007. AgenaRisk software package, www.AgenaRisk.com.

Agrawal, A. K., Kawaguchi, A. \& Chen, Z. 2009. Bridge element deterioration rates (No. C-01-51). New York State Department of Transportation.

Andrews, J. 2012. A modelling approach to railway track asset management. Proceedings of the Institution of Mechanical Engineers, Part F: Journal of Rail and Rapid Transit, 227(1), 56-73.

Audley, M. \& Andrews, J. A Petri-Net Modelling Approach to Rail Track Geometry Maintenance and Inspection. Advances in Risk \& Reliability Technology Symposium 2013, 230-243.

Cesare, M. A., Santamarina, C., Turkstra, C. \& Vanmarcke, E. H. 1992. Modeling bridge deterioration with Markov chains. Journal of Transportation Engineering, 118, 820833.

Dhillon, B. S. 2002. Engineering maintenance: a modern approach. CRC Press.
Edirisinghe, R., Setunge, S. \& Zhang, G. 2012. Application of Gamma process for deterioration prediction of buildings from discrete condition data. Sri Lankan Journal of Applied Statistics, 12

Frangopol, D. M., Kallen, M. J. \& Van Noortwijk, J. M. 2004. Probabilistic models for life-cycle performance of deteriorating structures: review and future directions. Progress in Structural Engineering and Materials, 6, 197-212.

Gorjian, N., Ma, L., Mittinty, M., Yarlagadda, P. \& Sun, Y. 2010. A review on degradation models in reliability analysis. Engineering Asset Lifecycle Management. Springer.

Guler, H., Jovanovic, S. \& Evren, G. Modelling railway track geometry deterioration. Proceedings of the Institution of Civil Engineers-Transport, 2011. Thomas Telford Ltd, 65-75.

He, Q., Li, H., Bhattacharjya, D., Parikh, D. P. \& Hampapur, A. Railway track geometry defect modeling: deterioration, derailment risk and optimal repair. Proceedings of the Transportation Research Board 92ndAnnual Meeting, 2013.

Jiang, Y., Saito, M. \& Sinha, K. C. 1988. Bridge performance prediction model using the Markov chain (No. 1180). Transportation Research Board.

Jun, C. H., Balamurali, S. \& Lee, S. H. 2006. Variables sampling plans for Weibull distributed lifetimes under sudden death testing. Reliability, IEEE Transactions on, $55,53-58$

Kumar, S. 2006. A study of the rail degradation process to predict rail breaks. Luleå University of Technology.

Le, B. \& Andrews, J. 2015. Modelling railway bridge degradation based on historical maintenance data. Advances in Risk \& Reliability Technology Symposium, Loughborough, 23rd - 25th June 2015.

Marquez, D., Neil, M. \& Fenton, N. 2007. A new Bayesian Network approach to Reliability modelling. Mathematical Methods in Reliability (MMR07), Jul 2007.

Marsh, D. W. R., Nur, K., Yet, B. \& Majumdar, A. 2015. Using operational data for decision making: a feasibility study in rail maintenance. Advances in Risk \& Reliability Technology Symposium, Loughborough, 23rd - 25th June 2015.

Micevski, T., Kuczera, G. \& Coombes, P. 2002. Markov model for storm water pipe deterioration. Journal of infrastructure systems, 8, 49-56.

Neil, M., Tailor, M. \& Marquez, D. 2007. Inference in hybrid Bayesian networks using dynamic discretization. Statistics and Computing, 17(3), pp.219-233.

Nordman, D. J. \& Meeker, W. Q. 2002. Weibull prediction intervals for a future number of failures. Technometrics, $44,15-23$

Pintelon, L. M. \& Gelders, L. 1992. Maintenance management decision making. European journal of operational research, 58, 301-317.

Rama, D. \& Andrews, J. A System-wide Modelling Approach to Railway Infrastructure Asset Management. Advances in Risk and Reliability Technology Symposium, 2013.

Shafahi, Y. \& Hakhamaneshi, R. 2009. Application of a maintenance management model for Iranian railways based on the Markov chain and probabilistic dynamic programming. International Journal of Science and Technology. Transaction A: Civil Engineering, 16, 87-97. 